\title{
Enquête sur les causes de mortalité des pintadeaux au Burkina Faso
}

\author{
R. Bessin ${ }^{1}$ A.M.G. Belem ${ }^{2}$ H. Boussini ${ }^{1}$ \\ Z. Compaore ${ }^{3}$ Y. Kaboret ${ }^{4}$ M.A. Dembele ${ }^{5}$
}

\section{Mots-clés}

Pintade - Numida meleagris - M ortalité Technique d'élevage - Facteur du milieu - Saison humide - Burkina Faso.

\section{Résumé}

Cette étude sur les causes de mortalité des pintadeaux, Numida meleagris, a comporté une enquête conduite dans huit provinces du Burkina Faso chez 114 éleveurs et une série d'études réalisées sur 58 élevages localisés sur une aire plus réduite de cinq provinces avec le suivi quotidien de 3017 pintadeaux âgés de 0 à 3 mois. L'enquête a révélé, d'une part, un taux global de mortalité de 73 p. 100 des pintadeaux et, d'autre part, de grandes insuffisances des techniques d'élevage de la pintade en matière d'habitat, de chauffage, d'alimentation, d'abreuvement et de santé. Au niveau du suivi quotidien de 3017 pintadeaux, il a été trouvé que les taux de mortalité étaient de l'ordre de 80 p. 100 dans les élevages améliorés et traditionnels et que la période de mortalité maximale se situait en août pendant la saison pluvieuse. Les germes isolés sur les élevages traditionnels ou améliorés étaient : Escherichia coli, Salmonella sp., Klebsiella sp., Enterobacter sp., Pseudomonas sp., Proteus sp. et Candida albicans. Q uant aux parasites, les types suivants ont été identifiés : des trichomonades, des coccidies, des ascaris, des ténias et des spirures du genre Tetrameres. Cette étude a montré que les causes de mortalité des pintadeaux au Burkina Faso étaient multifactorielles et que toute opération d'amélioration de la méléagriculture (production de pintade) devrait, en plus des conditions d'élevage, tenir compte des infections simultanées, de l'âge et de la saison.

\section{- INTRODUCTION}

Dans les pays africains soucieux de l'autosuffisance alimentaire, la carence en protéine animale est sérieuse, particulièrement pour les populations rurales. Pour cette population rurale africaine, la consommation de viande par an et par habitant est de $10 \mathrm{~kg}$ contre $200 \mathrm{~kg}$ de céréales (2).

Le développement, au Burkina Faso, des animaux de basse-cour et surtout de la pintade, Numida meleagris, connaît de nos jours un essor important. Ceci est lié à un contexte économique difficile en

\footnotetext{
1. Laboratoire National d'Elevage, 03 BP 7026, Ouagadougou 03, Burkina Faso 2. Université d'Ouagadougou, 01 BP 4626, Ouagadougou 01, Burkina Faso

3. Centre Régional de Promotion Agro-Pastorale du Sahel, Dori, Burkina Faso

4. Ecole Inter-Etats de Sciences et Médecine Vétérinaires (EISMV), BP 5077, Dakar, Sénégal

5. Projet de Développement des Animaux Villageois (PDAV), 01 BP 1907, Ouagadougou 01, Burkina Faso
}

particulier pour les populations rurales. L'élevage de la pintade fait l'objet d'une spéculation économique (4) stimulée par la récente dévaluation du franc CFA et constitue non seulement une source importante de revenus pour les paysans mais aussi de produits d'exportation. Avant la dévaluation, l'effectif des pintades au Burkina Faso était déjà estimé en 1989 à 2775000 têtes, représentant 15,6 p. 100 des effectifs de volailles $(2,5)$. La production de volailles du pays était alors évaluée à environ 15000 tonnes par an, toutes espèces confondues, dont 5000 tonnes étaient exportées en vif vers les pays côtiers voisins (Côte d'Ivoire, Ghana, Togo, Bénin) (5)

Cet élevage, courant dans l'ensemble du pays, se heurte à de nombreuses contraintes touchant principalement la santé, l'alimentation et l'habitat des pintadeaux. Les pathologies, encore mal connues et donc mal maîtrisées, entraînent des mortalités importantes, particulièrement chez les pintadeaux âgés de 0 à 3 mois. Au cours des dernières campagnes agricoles, ces mortalités, contre lesquelles aucune mesure efficace n'a pu être prise, ont constitué la contrainte majeure de la production de pintades. Cette étude a 
été initiée devant l'ampleur de ce problème afin de contribuer à identifier les causes de mortalité des pintadeaux, étape indispensable à la proposition d'un plan adéquat de prophylaxie et d'amélioration des techniques d'élevage.

\section{MATERIEL ET METHO DES}

Cette étude a été conduite en deux parties. La première a permis, à travers une enquête $(2,3,6)$, de préciser les taux de mortalité et les conditions d'élevage des pintades, au Burkina Faso. La deuxième partie a consisté à suivre quotidiennement des élevages de pintades pour identifier les agents jouant un rôle éventuel dans la mortalité des pintadeaux. Cette étude s'est déroulée de juillet 1994 à janvier 1995 .

\section{Enquête sur les taux de mortalité et les conditions d'élevage}

Au Burkina Faso, les zones éco-climatiques Nord et Sud-soudaniennes, situées respectivement sous les isohyètes 650 et $1000 \mathrm{~mm}$, sont les plus favorables à l'élevage de la pintade et ont les effectifs les plus élevés. Pour cette enquête, 114 éleveurs ont été contactés dans huit provinces de ces deux zones : le Bazega, le Boulgou, le Boulkiemde, le Houet, le Kadiogo, la Kossi, le Ganzourgou et l'Oubritenga (figure 1). Sur la base d'une méthode de recherche participative appelée rapid rural appraisal (RRA) ou « évaluation rapide en milieu rural » (2), un questionnaire détaillé sur différents aspects de la production de pintades a été établi et utilisé auprès des éleveurs. Outre les informations relatives à l'identification de l'éleveur et à la date de l'enquête, le questionnaire a concerné les types d'élevage (traditionnel ou amélioré) ou de poulaillers, le chauffage, l'hygiène de l'habitat, la nature, la fréquence et les périodes journalières de distribution des aliments, la nature et l'entretien des abreuvoirs et des mangeoires, la qualité de l'eau, les effectifs d'oiseaux, les mortalités et enfin les dominantes pathologiques observées ainsi que les traitements réalisés.

\section{Etude des causes de mortalité}

Ces études ont consisté en des suivis quotidiens d'élevages traditionnels ou améliorés de pintades, au cours desquels ont été réalisés des observations cliniques, des autopsies et des examens de laboratoire. Ces suivis ont touché une zone et des effectifs d'éleveurs et de pintades plus réduits que ceux des enquêtes.

\section{Zone d'étude}

Quatre sites ont été retenus dans la zone Nord-soudanienne (Ouagadougou dans la province du Kadiogo, Sabou dans le Boulkiemde, Tenkodogo dans le Boulgou, Ziniare dans l'Oubritenga) et un seul site dans la zone Sud-soudanienne (Bereba dans la province du Houet) (figure 1). Ces sites, situés dans cinq provinces au total, ont été choisis en fonction des facilités de travail et de l'existence de deux types d'élevage : traditionnel et amélioré. Dans l'élevage traditionnel, la pintade ne reçoit de la part du propriétaire que des soins de protection contre les prédateurs et

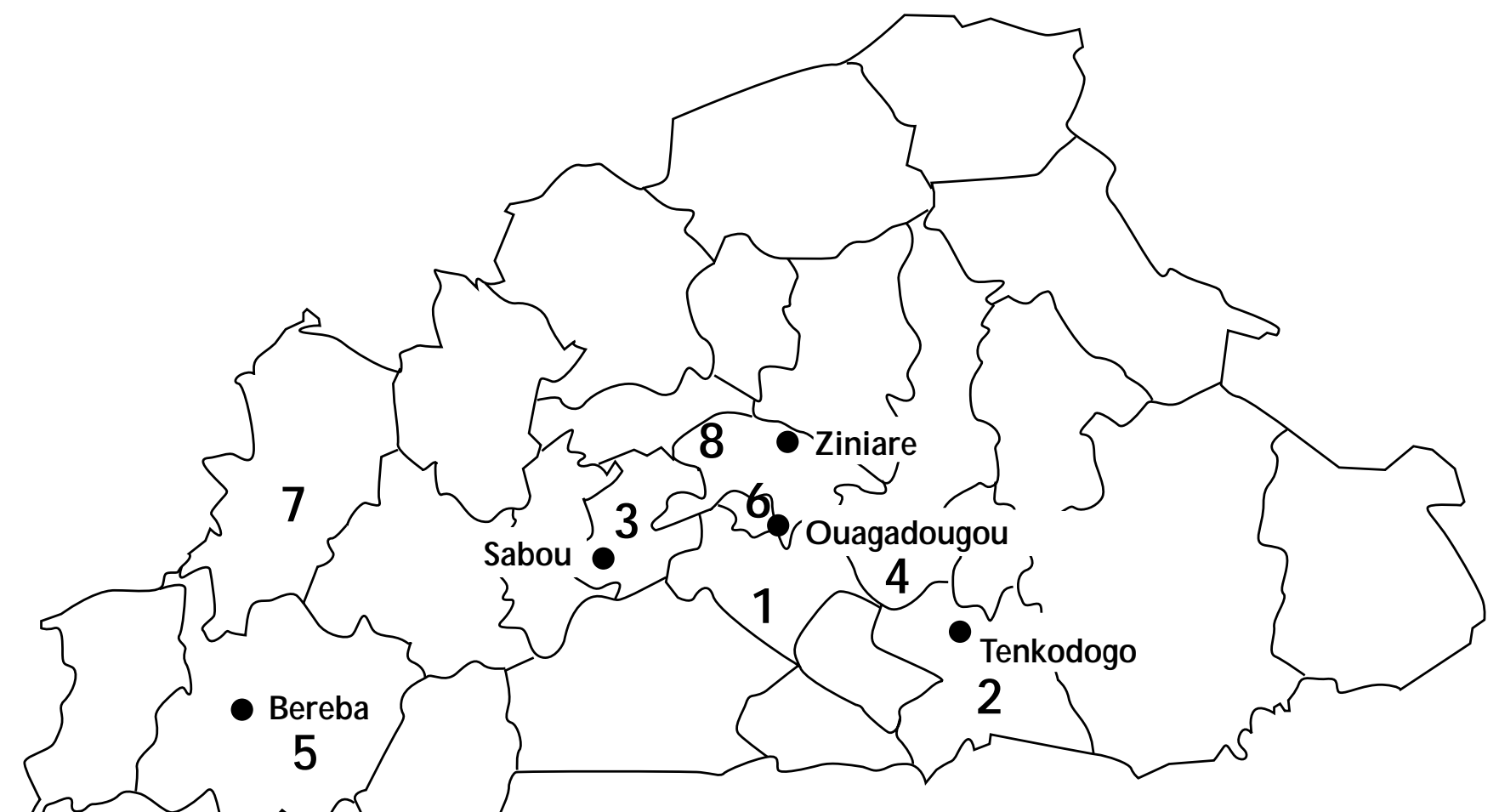

Provinces couvertes par les enquêtes :

1. Bazega

2. Boulgou

3. Boulkiemde

4. Ganzourgou

5. Houet

6. Kadiogo

7. Kossi

8. 0 ubritenga

Sites concernés par les suivis quotidiens

Figure 1 : provinces du Burkina Faso concernées par l'étude. 
quelques dangers naturels tels que les flaques d'eau. Dans l'élevage amélioré, les pintades disposent en plus d'abreuvoirs d'un aliment complet de démarrage pour poussins et de lampes tempêtes pour le chauffage.

\section{Effectifs des oiseaux étudiés}

Le tableau I présente le nombre d'éleveurs et les effectifs des pintadeaux par site concernés par les suivis quotidiens. La plupart des pintadeaux ont été obtenus par couvaison des œufs par des poulesmères, excepté les deux élevages améliorés d'Ouagadougou et de Tenkodogo où les pintadeaux ont été incubés artificiellement. Tous les pintadeaux suivis étaient âgés de 0 à 3 mois

Sur les 3017 pintadeaux suivis dans 58 élevages au total, 1186 provenaient d'élevages traditionnels et 1831 d'élevages améliorés. Il faut noter que les oiseaux étudiés n'ont jamais été vaccinés ou déparasités, sauf dans quelques rares élevages améliorés de Bereba dans la province du Houet.

\section{Enquêtes anatomo-cliniques et examens de laboratoire}

Une équipe de deux enquêteurs au moins par site assurait quotidiennement le suivi des 58 élevages faisant partie de l'étude. Des fiches d'enquête ont été utilisées pour le suivi clinique, les commémoratifs des pathologies observées et le recensement des cas de mortalité. Les cadavres étaient récupérés dans des sacs en plastique, scellés et envoyés dans les $24 \mathrm{~h}$ sous froid au Laboratoire national d'élevage pour autopsie et examens complémentaires $(1,8)$

Les autopsies ont été réalisées, lors des suivis quotidiens, sur des cadavres et des pintadeaux malades sacrifiés pour les besoins de l'étude. Ils provenaient tous des quatre provinces suivantes : le Boulgou, le Boulkiemde, le Kadiogo et l'Oubritenga. La province du Houet n'a pu être concernée par les autopsies suite à des difficultés d'acheminement rapide des sujets au Laboratoire national d'élevage. Suivant les lésions, des prélèvements de foie, cœur, poumon, rate et rein ont été faits pour des examens bactériologiques. Ils ont été ensemencés sur des milieux classiques de culture et les germes identifiés à l'aide de galeries. Les examens parasitologiques ont été effectués sur des matières fécales ou les contenus du cæcum, des intestins, et d'autres parties du tube digestif. Les parasites gastro-intestinaux furent recherchés par examen microscopique direct ou par autopsie helminthologique de tube digestif pour la collecte de parasites immatures ou adultes.

\section{Tableau I}

Répartition des effectifs de pintadeaux concernés par les suivis quotidiens en fonction du type d'élevage et du site

\begin{tabular}{llrr} 
Types d'élevage & Sites & $\begin{array}{c}\text { Nb. } \\
\text { d'éleveurs }\end{array}$ & Effectifs \\
\hline Traditionnel & Bereba & 7 & 273 \\
& Sabou & 10 & 498 \\
& Ziniare & 8 & 415 \\
Amélioré & Bereba & 10 & 410 \\
& Sabou & 10 & 509 \\
& Ziniare & 10 & 380 \\
& Ouagadougou & 2 & 249 \\
& Tenkodogo & 1 & 283 \\
Total & 5 & 58 & 3017
\end{tabular}

Les anticorps inhibant l'hémagglutination ont été recherchés pour la détection du virus de la maladie de Newcastle par la technique sérologique du laboratoire de pathologie aviaire de Ploufragran (11). Ces tests ont été réalisés sur deux séries de sérums récoltés sur les mêmes groupes de pintadeaux de deux semaines à un mois d'intervalle. La première série de sérums a été prélevée sur des pintadeaux d'environ un mois d'âge. Les provinces du Boulgou, du Houet et du Kadiogo disposant d'un laboratoire avec une centrifugeuse et un congélateur ont été retenues comme sites pour ces examens sérologiques. Les sérums ont été récoltés à partir de sang prélevé à l'aide de seringues de $5 \mathrm{ml}$ par ponction des veines jugulaires droites des pintadeaux. Ils ont été conservés au congélateur à $-20{ }^{\circ} \mathrm{C}$ jusqu'au moment de leur utilisation. Le titre de chaque sérum a été donné par l'inverse de la dernière dilution présentant encore une inhibition totale de l'hémagglutination. Un titre inférieur à 20 était considéré négatif et un titre supérieur ou égal à 40 positif pour le virus de la maladie de Newcastle.

\section{RESU LTATS}

\section{Taux de mortalité et conditions d'élevage des pintadeaux}

Dix à 20 éleveurs par province, possédant ensemble de 643 à 2364 pintadeaux, ont été concernés par le questionnaire lors des enquêtes couvrant les huit provinces. Le tableau II résultant de ces enquêtes montre les taux de mortalité par province et le taux global (73 p. 100) pour toute la zone des huit provinces enquêtées.

Quant aux taux de mortalité calculés lors du suivi de 3017 pintadeaux appartenant à 58 élevages différents de cinq provinces du pays, ils étaient de 80 p. 100 pour les élevages améliorés ou traditionnels. De plus, au niveau des élevages suivis, les mortalités étaient maximales en août, mois le plus humide de la saison pluvieuse (figure 2).

Les fiches d'enquête sur les huit provinces ont permis de préciser de nombreux aspects touchant les conditions d'élevage. Parmi les élevages, 9 p. 100 étaient en totale liberté alors que le reste utilisait une forme d'habitat soit de type traditionnel en banco ou en paille (80 p. 100), soit de type amélioré confectionné en parpaings avec une hauteur convenable et l'intérieur avec des murs crépis (9) (11 p. 100).

\section{Tableau II}

Résultats de l'enquête sur les taux de mortalité des pintadeaux (0-3 mois d'âge) au niveau des huit provinces

\begin{tabular}{lccc} 
Provinces & $\begin{array}{c}\text { Nb. de } \\
\text { Producteurs }\end{array}$ & $\begin{array}{c}\text { Effectif des } \\
\text { pintadeaux } \\
\text { enquétés }\end{array}$ & $\begin{array}{c}\text { Mortalité } \\
(\%)\end{array}$ \\
\hline Bazega & 10 & 738 & 77 \\
Boulgou & 18 & 2364 & 67 \\
Boulkiemde & 20 & 2303 & 69 \\
Ganzourgou & 12 & 831 & 89 \\
Houet & 19 & 1951 & 63 \\
Kossi & 10 & 820 & 82 \\
Kadiogo & 10 & 643 & 76 \\
Oubritenga & 15 & 1426 & 84 \\
Total & 114 & 11076 & 73
\end{tabular}


Seulement 4 p. 100 des éleveurs utilisaient des lampes tempêtes pour chauffer les pintadeaux alors que les autres se contentaient du comportement maternel des poules couveuses. Trente-neuf pour cent des poulaillers étaient nettoyés une ou deux fois par an au maximum. Pour ce nettoyage, des désinfectants n'étaient utilisés que dans un cas sur 100.

Les aliments distribués aux pintadeaux au démarrage étaient de natures diverses : mil, maïs, sorgho ou termites dans 87 p. 100 des élevages et un aliment complet pour poussins dans 5 p. 100 des cas. Dans les autres élevages (8 p. 100), du son de céréales, des restes de cuisine, du sésame ou de la drêche de dolo (bière locale) étaient utilisés. Alors que 16 p. 100 des éleveurs ne distribuaient l'aliment qu'une fois par jour, 54 p. 100 le faisaient plus de trois fois.

Différents matériaux sont utilisés pour la confection des abreuvoirs. Les abreuvoirs de type CNA (Centre national avicole) fabriqués en terre cuite et beaucoup plus adaptés pour l'aviculture étaient utilisés par seulement 30 p. 100 des éleveurs. Des morceaux de canaris cassés étaient utilisés par 53 p. 100 des éleveurs alors que dans 17 p. 100 des cas les abreuvoirs étaient faits d'assiettes, de bois taillé et de bidons usagés. Chez 4 p. 100 des éleveurs ces abreuvoirs n'étaient jamais nettoyés, ils l'étaient quotidiennement ou de manière hebdomadaire dans respectivement 30 et 10 p. 100 des élevages, et irrégulièrement dans 56 p. 100 des élevages restants. L'eau d'abreuvement provenait de puits (70 p. 100), de forages (19 p. 100) et de mares, de rivières ou de barrages (11 p. 100).

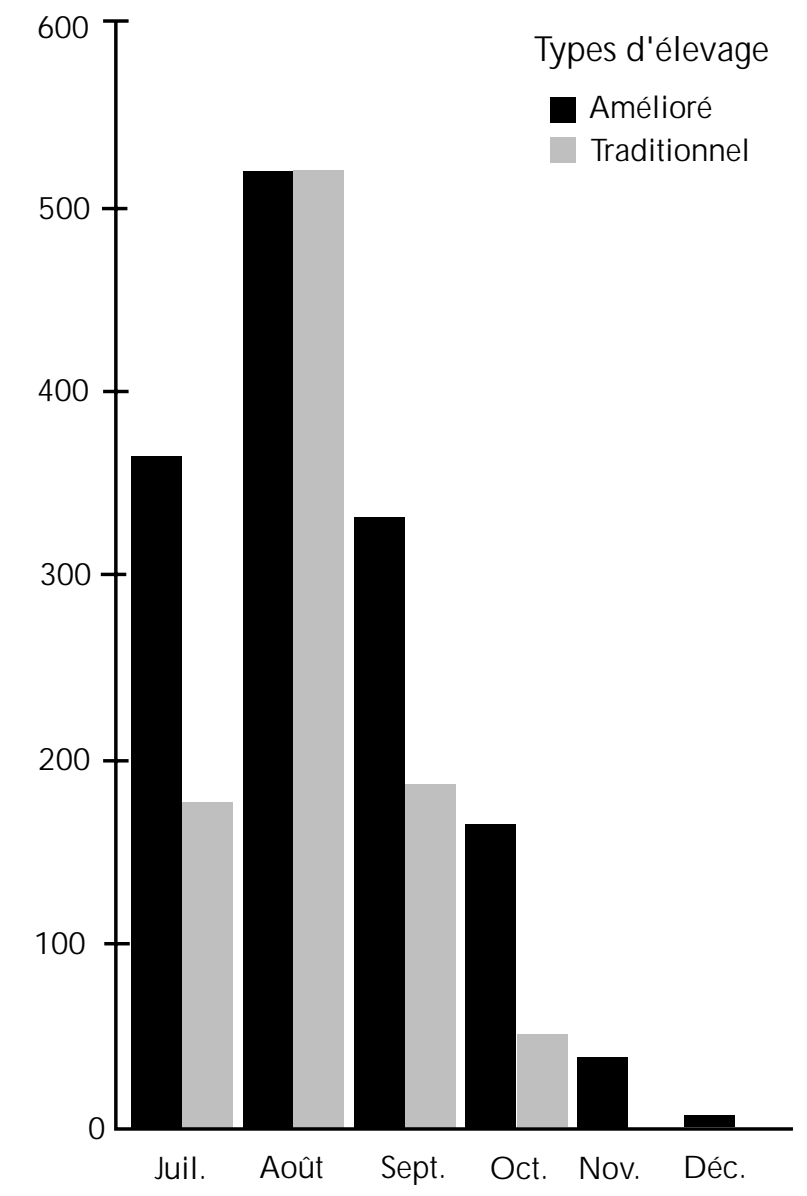

Figure 2 : répartition des effectifs de pintadeaux morts par mois selon le type d'élevage au niveau des 58 élevages suivis quotidiennement.

\section{Examens anatomo-cliniques}

Les résultats des examens cliniques provenaient des suivis quotidiens de 3017 pintadeaux chez 58 éleveurs dans cinq provinces. Les symptômes, lésions et évolutions des cas morbides estimés à 2469 pintadeaux étaient souvent similaires dans la plupart des élevages. L'abattement, la somnolence et la diarrhée étaient souvent observés. Dans les formes suraiguës ou aiguës évoluant rapidement vers la mort en $24 \mathrm{~h}$, les pintadeaux étaient extrêmement frileux et présentaient des plumes ébouriffées et des ailes pendantes (les éleveurs disent que la pintade porte un grand « boubou »). Ils refusaient de s'alimenter et de s'abreuver et s'entassaient autour d'une source de chaleur quand elle était disponible. Dans les formes d'évolution plus lentes, les symptômes cités s'accompagnaient de cris plaintifs, d'une respiration difficile, d'une démarche chancelante avec en fin d'évolution la paralysie et la mort en 48 à $72 \mathrm{~h}$. Des signes cliniques moins constants observés dans les formes d'évolution lente étaient la déshydratation extrême avec une sécheresse des pattes et des appendices céphaliques et la kérato-conjonctivite unie ou bilatérale souvent opacifiante. Les symptômes observés n'ont pas permis d'identifier des maladies précises.

Au total 305 pintadeaux malades, déjà morts ou sacrifiés ont été autopsiés. Les résultats sont consignés dans le tableau III. Les organes les plus fréquemment atteints de lésions étaient d'abord le foie, puis les intestins et le cæcum, enfin les organes du système cardio-respiratoire.

\section{Examens bactériologiques et parasitologiques}

Parmi les 305 autopsies de pintadeaux, 217 et 88 provenaient respectivement d'élevages de type amélioré ou traditionnel. Respectivement 80 et 73 p. 100 des prélèvements se sont révélés positifs pour au moins un microorganisme. Les microorganismes suivants ont été isolés : Escherichia coli, Salmonella sp., Klebsiella sp., Enterobacter sp., Pseudomonas sp., Proteus sp. et Candida albicans (tableau IV).

Les examens parasitologiques ont été réalisés sur tous les prélèvements provenant des autopsies. Les prévalences des infestations calculées étaient de 85 et 76 p. 100 respectivement en élevages améliorés et traditionnels. Les types de parasites suivants ont été identifiés dans les prélèvements : trichomonades, ookystes de coccidies, ascaris, ténia et spirures du genre Tetrameres (tableau V). Les genres et espèces de tous les parasites n'ont pas pu être identifiés. Pour les parasites du genre Tetrameres et les ténias, les prévalences étaient respectivement de 0 et 1 p. 100 (un seul pintadeau positif) en élevage amélioré. Par contre, en élevage traditionnel, elles étaient respectivement de 3 et 20 p. 100. Les taux d'infection par des ookystes de coccidies, ascaris et Trichomonas sp. étaient plus importants dans les élevages améliorés que dans les élevages traditionnels. Il faut également signaler qu'en élevages améliorés et traditionnels respectivement 2 et 3 p. 100 des prélèvements présentaient des cristaux d'oxalate de chaux.

\section{Examens sérologiques}

Au cours de deux périodes séparées de deux semaines à un mois, 625 sérums ont été récoltés sur les mêmes groupes d'oiseaux. Ils ont été soumis au test de détection des anticorps anti maladie de Newcastle. Au niveau des élevages améliorés et traditionnels, respectivement 150 et 145 sérums ont été analysés pour la première période de prélèvement puis 167 et 163 pour la deuxième période. A la première prise de sang, les prévalences de sérums positifs étaient de 1 et 7 p. 100 respectivement en élevages améliorés et traditionnels alors qu'à la deuxième prise de sang, ils étaient de 0 et 20 p. 100. 
Tableau III

Principales lésions observées lors de 305 autopsies

\begin{tabular}{|c|c|c|c|}
\hline 0 rganes & $\begin{array}{l}\text { Types } \\
\text { de lésions }\end{array}$ & $\begin{array}{c}\text { N b. } \\
\text { d'organes }\end{array}$ & $\begin{array}{c}\% \\
\text { de } \\
\text { pintadeaux }\end{array}$ \\
\hline $\begin{array}{l}\text { Intestin } \\
\text { et cæcum }\end{array}$ & Inflammation & 244 & 80 \\
\hline $\begin{array}{l}\text { Proventricule } \\
\text { et gésier }\end{array}$ & $\begin{array}{l}\text { Inflammation, } \\
\text { atrophie } \\
\text { et hypertrophie }\end{array}$ & 96 & 31 \\
\hline Cloaque & Hypertrophie & 46 & 15 \\
\hline Foie & $\begin{array}{l}\text { Inflammation } \\
\text { et décoloration }\end{array}$ & 245 & 80 \\
\hline $\begin{array}{l}\text { Rate et } \\
\text { pancréas }\end{array}$ & $\begin{array}{l}\text { Hypertrophie, } \\
\text { décoloration } \\
\text { et inflammation }\end{array}$ & 50 & 16 \\
\hline $\begin{array}{l}\text { Sinus, } \\
\text { sacs aériens } \\
\text { et poumons }\end{array}$ & $\begin{array}{l}\text { Inflammation, } \\
\text { décoloration } \\
\text { et nodules }\end{array}$ & 175 & 57 \\
\hline Cœur & $\begin{array}{l}\text { Inflammation } \\
\text { et adhérences }\end{array}$ & 147 & 48 \\
\hline Rein & Inflammation & 83 & 27 \\
\hline $\begin{array}{l}\text { Bourse de } \\
\text { Fabricius }\end{array}$ & $\begin{array}{l}\text { Hypertrophie } \\
\text { et dépôt } \\
\text { de caséum } \\
\text { souvent calcifié }\end{array}$ & 60 & 20 \\
\hline Muscles & Inflammation & 70 & 23 \\
\hline $\begin{array}{l}\text { Yeux et } \\
\text { appendices } \\
\text { céphaliques }\end{array}$ & $\begin{array}{l}\text { Kérato-conjonctivite } \\
\text { avec opacification } \\
\text { et lésions } \\
\text { varioliques }\end{array}$ & 75 & 25 \\
\hline Pattes & $\begin{array}{l}\text { Paralysie, } \\
\text { malformation } \\
\text { et déformation }\end{array}$ & 10 & 3 \\
\hline
\end{tabular}

\section{DISCUSSIO N}

Cette étude a révélé un taux de mortalité de 73 p. 100 lors des enquêtes et, lors des suivis quotidiens, des taux de mortalité de 80 p. 100 au Burkina Faso que ce soit en élevage amélioré ou traditionnel de pintadeaux. La méthode de recherche participative RRA suivie pour les enquêtes a donné des taux de mortalité légèrement plus faibles mais elle s'est néanmoins montrée complémentaire de la méthode de suivi quotidien pour la récolte des informations de base concernant la production de pintades. Le suivi quotidien d'élevages de pintades a permis plus particulièrement de montrer que le pic de mortalité des jeunes pintadeaux se situait au mois d'août, période la plus humide de l'année. La coïncidence entre le pic de mortalité et la saison de reproduction des pintades (9), en août quand les effectifs sont les plus importants, compromet sérieusement les potentialités de cet élevage.

Selon les observations réalisées lors des enquêtes, les causes de mortalité en élevage traditionnel aussi bien qu'amélioré peuvent être multifactorielles et concerner plusieurs aspects de la production de la pintade : l'habitat, l'alimentation, l'abreuvement et la santé. Si la plupart des élevages se faisaient dans des habitats (91 p. 100), il faut néanmoins signaler que ces poulaillers étaient en très mauvais état, peu aérés, mal entretenus et n'étaient presque jamais nettoyés. Les poulaillers améliorés, confectionnés en parpaings avec un intérieur crépi et une hauteur convenable, mis au point par le PDAV, n'étaient utilisés que par 11 p. 100 des éleveurs et étaient souvent mal entretenus. L'utilisation d'un habitat convenable pour les pintadeaux contribuerait certainement à les protéger des pluies torrentielles (fréquentes au mois d'août) et des prédateurs. La nécessité de réchauffer les pintadeaux pendant les premiers jours de leur vie est mal connue des éleveurs (seulement 4 p. 100 d'entre eux utilisaient des lampes tempêtes).

Tous les éleveurs distribuaient des aliments aux pintadeaux mais en quantité et qualité insuffisantes. Cet apport alimentaire avait pour principal objectif de domestiquer les oiseaux et de les maintenir dans les concessions. L'eau distribuée aux pintades provenait dans 90 p. 100 des cas de puits et forages, plus propres que les mares, les rivières ou les barrages contenant de l'eau sale et boueuse. Cependant, les abreuvoirs étaient insuffisamment nettoyés et étaient à la disposition de tous les autres animaux, domestiques ou non, des concessions.

Devant la médiocrité des résultats présentés ci-dessus concernant l'habitat, l'alimentation et l'abreuvement de la pintade, il s'avère indispensable non seulement de favoriser l'accès au crédit mais aussi d'organiser pour les éleveurs de pintades des séances de sen-

Tableau IV

Nombre et pourcentage de prélèvements positifs pour des microorganismes en fonction du type d'élevage

\begin{tabular}{lccccccccc}
$\begin{array}{l}\text { Types } \\
\text { d'elevage }\end{array}$ & $\begin{array}{c}\text { Nb. de } \\
\text { prélèvements }\end{array}$ & $\begin{array}{c}\text { Esche- } \\
\text { richia } \\
\text { coli }\end{array}$ & $\begin{array}{c}\text { Salmo- } \\
\text { nella } \\
\text { sp. }\end{array}$ & $\begin{array}{c}\text { Kleb- } \\
\text { siella } \\
\text { sp. }\end{array}$ & $\begin{array}{c}\text { Entero- } \\
\text { bacter } \\
\text { sp. }\end{array}$ & $\begin{array}{c}\text { Pseudo- } \\
\text { monas } \\
\text { sp. }\end{array}$ & $\begin{array}{c}\text { Proteus } \\
\text { sp. }\end{array}$ & $\begin{array}{c}\text { Candida } \\
\text { albicans }\end{array}$ & $\begin{array}{c}\text { Total } \\
\text { négatif } \\
(\%)\end{array}$ \\
\hline Amélioré & 217 & 29 & 8 & 6 & 4 & 10 & 23 & 14 & 20 \\
Traditionnel & 88 & 24 & 23 & 2 & 6 & 8 & 10 & 0 & 27
\end{tabular}


Tableau V

Nombre et pourcentage de prélèvements positifs pour des parasites en fonction du type d'élevage

\begin{tabular}{lccccccc}
$\begin{array}{l}\text { Types } \\
\text { d'élevage }\end{array}$ & $\begin{array}{c}\text { N b. de } \\
\text { prélèvements }\end{array}$ & $\begin{array}{c}\text { Trichomonas } \\
\text { sp. }\end{array}$ & Coccidie & Ascaris & Ténia & $\begin{array}{c}\text { Parasites isolés (\%) } \\
\text { Tetrameres }\end{array}$ & $\begin{array}{c}\text { Total } \\
\text { négatif } \\
(\%)\end{array}$ \\
\hline Amélioré & 217 & 14 & 65 & 18 & 1 & 0 & 15 \\
Traditionnel & 88 & 6 & 48 & 10 & 20 & 3 & 24
\end{tabular}

sibilisation, de formation et de vulgarisation des techniques simples sur l'élevage de la pintade (2). Des auteurs ont signalé qu'un contrôle rigoureux du développement de la pintade entre 0 et 6 semaines doit aboutir à une croissance satisfaisante et à un rendement optimal de l'oiseau adulte (2).

Les mortalités observées lors du suivi quotidien de 3017 pintadeaux sur cinq provinces n'ont pu être attribuées à aucun microorganisme ou parasite précis. Comme cela a déjà été signalé par Saunders (9), cette étude a montré qu'en règle générale les examens de laboratoire ont pu révéler la présence de plusieurs agents pathogènes. Les autopsies ont mis en évidence la présence très fréquente d'organes atteints. Il s'agit, par ordre de décroissance, du foie, de certaines parties du tube digestif telles que l'intestin, le cæcum et le complexe gésier-proventricule, d'organes de l'appareil cardio-respiratoire. En fonction de la fréquence des agents pathogènes isolés et identifiés, on peut considérer comme dominantes les pathologies suivantes : la colibacillose, la salmonellose, les helminthoses, la trichomonose et la coccidiose, alors que la candidose et la maladie de Newcastle étaient plutôt rares. Les trichomonades, ayant provoqué en 1980 au Burkina Faso des épizooties meurtrières dans les élevages de pintades (10) avec un taux global de mortalité de 88 p. 100 , ont été retrouvés dans cette étude en troisième position après les coccidies et les ascaris parmi les agents parasitaires isolés. Les conditions qui entraînent l'explosion meurtrière de la trichomonose gagneraient donc à être élucidées.

Cette étude confirme les observations de Saunders (9) sur l'importance des salmonelles au niveau surtout des élevages traditionnels. Dans ces élevages, 23 p. 100 des prélèvements ensemencés étaient positifs pour des salmonelles. Le Coz-Douin (7) a signalé qu'il existe plus de 2000 sérovars de salmonelles répertoriés à l'heure actuelle. Si certains sont adaptés aux volailles d'autres, par contre, peuvent infecter et être transmis entre tous les animaux, l'homme y compris. Il serait indispensable dans les études à venir de poursuivre toute identification de salmonelle isolée par la recherche de sérovar. Cela permettrait, non seulement une meilleure connaissance épidémiologique du germe, mais aussi une orientation plus sérieuse du pronostic et le succès du traitement.

Il faut enfin noter que les élevages de pintades enquêtés ne disposaient d'aucun programme de prophylaxie ou de suivi sanitaire, bien que certains éleveurs aient utilisé la pharmacopée traditionnelle sur leurs oiseaux à titre curatif. Des décoctions d'écorces et de racines de plantes (néré, caillcédrat, cerisier, baobab et autres) sont utilisées contre certaines maladies infectieuses, la potasse et le piment contre les diarrhées, la cendre de bois et les résidus de beurre de karité contre les parasites externes. Ces connaissances empiriques gagneraient bien sûr à être confirmées, expérimentées et éventuellement standardisées.

\section{CONCLUSION}

Au Burkina Faso, la méléagriculture se pratique essentiellement selon un mode traditionnel. La volaille en général fait l'objet de très peu d'attention et se présente comme un produit de cueillette, vite récolté en cas de besoin. Une amélioration des principaux aspects de la production des pintades contribuerait à diminuer les taux de mortalité élevés de ces oiseaux et en conséquence à améliorer l'alimentation des populations. Les causes de mortalité des pintadeaux au Burkina Faso sont multifactorielles et impliquent probablement autant les conditions d'élevage et l'alimentation que des microorganismes et des parasites.

\section{BIBLIO GRAPHIE}

1. ALAMARGO T.J., 1982. Manuel d'anatomie et d'autopsie aviaire. $M$ aisons-Alfort, France, Le point vétérinaire, $48 \mathrm{p}$.

2. Atelier régional sur le développement de l'élevage de la pintade en régions sèches africaines, vol. 1 et 2, 1992. Rome, Italie, FAO, 125 p.

3. BARNOUIN J., FAYET J.C., JAY M., BROCHART M., 1986. Enquête écopathologique continue : facteurs de risque des mammites de la vache laitière. I. Analyses multidimensionnelles sur données d'élevage. Can. vet. J., $27:$ 135-145.

4. Développement de I'aviculture traditionnelle en Afrique tropicale, 1989. Fiches techniques d'élevage tropical. Maisons-Alfort, France, IEM VT-CIRAD, 8 p.

5. Enquête nationale sur l'effectif du cheptel, 1989. O uagadougou, Burkina Faso, Secrétariat d'Etat à l'élevage, 139 p.

6. FAYE B., LEFEVRE P.C., LANCELOT R., QUIRIN R., 1994. Ecopathologie animale : méthodologie, applications en milieu tropical. M aisons-Alfort, France, CIRAD-EMVT, 119 p.

7. Le COZ-DOUIN J., 1992. L'élevage de la pintade. Maisons-Alfort, France, Le point vétérinaire, $46 \mathrm{p}$.

8. LE TURDU Y., 1988. L'autopsie, le prélèvement, les commémoratifs. In : Aviculture française. Paris, France, Ministère de l'agriculture, p. 477-482.

9. SAUNDERS M.J., 1984. Aviculture traditionnelle en Haute-Volta : synthèse des connaissances actuelles et réflexions autour d'une expérience de développement (1979-1984), tomes I et II. O uagadougou, Burkina Faso, Ministère du développement rural, $483 \mathrm{p}$

10. SAUNDERS M.J., 1984. La trichomonose de la pintade en HauteVolta : une protozoose meurtrière et méconnue. Projet de Développement de l'aviculture villageoise. O uagadougou, Burkina Faso, Ministère du Développement rural, $11 \mathrm{p}$

11. Techniques pour la réalisation des tests d'hémagglutination et d'inhibition de l'hémagglutination, 1987. Plouflagran, France, Laboratoire national de pathologie aviaire, $4 \mathrm{p}$.

Reçu le 16.9.96, accepté le 3.4 .98 


\section{Summary}

Bessin R., Belem A.M.G., Boussini H., Compaore Z., Kaboret Y., Dembele M.A. Causes of young guinea fowl mortality in Burkina Faso

To investigate the causes of young guinea fow (N umida meleagris) mortality a survey involving 114 farmers in eight provinces was conducted in Burkina Faso, and a series of studies were carried out in 58 farms located in a more restricted area consisting of five provinces where 3017 guinea fowls 0-3 months of age were monitored daily. The survey showed on one hand a total mortality rate of $73 \%$ and on the other hand deficient rearing techniques concerning habitat, heating, nutrition, water and health. Daily monitoring of 3017 young fowls showed that mortality rates were estimated at $80 \%$ in improved and traditional farms, and the highest mortality rate was observed in August during the rainy season. In traditional or improved farms the microorganisms identified were Escherichia coli, Salmonella sp., Klebsiella sp., Enterobacter sp., Pseudomonas sp., Proteus sp. and Candida albicans. The parasites were trichomonads, coccidia, Ascaris, tapeworms, and spirurides of the genus Tetrameres. This study showed that there were many causes for young guinea fow 1 mortality in Burkina Faso. Moreover, farming conditions, as well as simultaneous infections, age and season have to be taken into account to improve guinea fowl farming.

Key words: Guinea fowl - Numida meleagris - Mortality Rearing technique - Environmental factor - Wet season Burkina Faso.

\section{Resumen}

Bessin R., Belem A.M.G., Boussini H., Compaore Z., Kaboret Y., Dembele M.A. Encuesta sobre las causas de mortalidad de los pollos de pintada en Burkina Faso

El presente estudio sobre las causas de mortalidad de los pollos de pintada, Numida meleagris, se basó en una encuesta llevada a cabo en 8 provincias de Burkina Faso, con 114 criadores y una serie de estudios realizados en 58 criaderos localizados en un área más reducida, de 5 provincias, con el seguimiento diario de 3017 pollos de pintada de 0 a 3 meses de edad. La encuesta demostró, por un lado, una tasa global de mortalidad de $73 \%$ de los pollos y por otra parte, enormes insuficiencias técnicas en la crianza del pollo de pintada en materia de habitat, calefacción, alimentación, suministro de agua y de salud. A nivel del seguimiento cotidiano de 3017 pollos de pintada, se encontró que las tasas de mortalidad fueron del orden de $80 \%$ en los criadores mejorados y tradicionales, con un período de mortalidad máximo situado en agosto, durante la estación de las Iluvias. Los gérmenes aislados en los criaderos tradicionales o mejorados fueron: Escherichia coli, Salmonella sp., Klebsiella sp., Enterobacter sp., Pseudomonas sp., Proteus sp. y Candida albicans. En cuanto a los parásitos, se identificaron los siguientes tipos: tricomonas, coccidias, áscaris, tenias y espiros del género Tetrameres. Este estudio demostró que las causas de mortalidad de los pollos de pintada en Burkina Faso son multifactoriales y que cualquier operación de mejoramiento de la meleagricultura (producción de pollos de pintada) debería tomar en cuenta, además de las condiciones de crianza, las infecciones simultáneas, la edad y la estación.

Palabras clave: Gallina de guinea - Numida meleagris M ortalidad - Técnica de crianza - Factor ambiental - Estación húmeda - Burkina Faso. 